\title{
Análisis de la rentabilidad de la producción de Flor de Jamaica (Hibiscus Sabdariffa)
}

\author{
Ignacio Caamal Cauich \\ Universidad Autónoma Chapingo \\ México \\ José Felix García Rodríguez \\ Universidad Juárez Autónoma de Tabasco \\ México \\ Verna Gricel Pat Fernández \\ Universidad Autónoma Chapingo \\ México \\ Vianey Lorenzo Ambrosio \\ Universidad Autónoma Chapingo \\ México
}




\title{
Análisis de la rentabilidad de la producción de Flor de Jamaica (Hibiscus Sabdariffa)
}

\author{
Ignacio Caamal Cauich ${ }^{1}\left(\mathbb{D}\right.$, José Felix García Rodríguez ${ }^{2}{ }^{\circledR}$, Verna Gricel Pat Fernández ${ }^{1}$ \\ (i) $\mathbf{y}$ Vianey Lorenzo Ambrosio ${ }^{1}$ \\ 1 Universidad Autónoma Chapingo, México \\ 2 Universidad Juárez Autónoma de Tabasco, México
}

Para citaciones: Caamal, I., García, J., Pat, V. y Ambrosio, V. (2020). Análisis de la rentabilidad de la producción de Flor de Jamaica (Hibiscus Sabdariffa). Panorama Económico, 28(2), 94-101.

https://doi.org/10.32997/pe-2020-2691

Recibido: 09/10/2019

Aprobado: 27/02/2020

Autor de correspondencia:

Ignacio Caamal Cauich

icaamal82@yahoo.com.mx

Editor: Andrés Escobar Espinoza Universidad de Cartagena, Colombia

Tipología IBN Publindex: Artículo de investigación científica y tecnológica

Copyright: @ 2020. Caamal, I., García, J., Pat, V. y Ambrosio, V. Este es un artículo de acceso abierto, distribuido bajo los términos de la licenciahttps://creativecommons.org/licenses/ by-nc-sa/4.0/ la cual permite el uso sin restricciones, distribución y reproducción en cualquier medio, siempre y cuando que el original, el autor y la fuente sean acreditados.

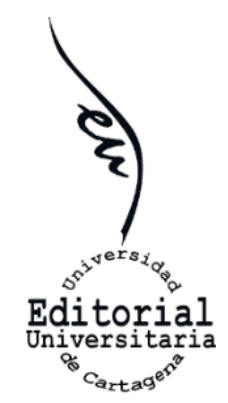

\section{RESUMEN}

La flor de Jamaica (Hibiscus Sabdariffa) es un cultivo que tiene propiedades terapéuticas, medicinales y nutricionales, y es originaria de África tropical. Los principales estados productores de flor de Jamaica en México son Guerrero y Oaxaca. El presente documento analiza los costos de producción, los ingresos y los indicadores de rentabilidad del cultivo de la flor de Jamaica en el municipio de Santo Domingo de Morelos del estado de Oaxaca (México). Se utilizó la metodología para la realización de estudios de costos y rentabilidad agrícola. Se encuentra evidencia sobre los indicadores financieros analizados: el valor de la relación beneficio-costo es mayor a uno, con una ganancia mayor a cero y el valor obtenido para el punto de equilibrio fue menor a la producción obtenida. Los resultados obtenidos permiten concluir que el cultivo de flor de Jamaica es rentable dadas sus características de producción en la zona.

Palabras clave: Costos; rendimiento; ganancias; competitividad.

JEL: M11, M19, M21

\section{Cost-Benefit analysis of Roselle (Hibiscus Sabdariffa) production}

\section{ABSTRACT}

Roselle (Hibiscus Sabdariffa) is a crop, from Tropical Africa, that has therapeutic, medicinal and nutritional properties. The main production of Roselle in Mexico takes place in the states of Guerrero and Oaxaca. This document analyses the production costs, income and profitability indicators of Roselle cultivation in the municipality of Santo Domingo de Morelos in the state of Oaxaca (Mexico). A methodology based on cost-benefit analysis and profitability was used during the research process. The evidence found as a result of the financial indicators analyzed shows that the value of the benefit-cost ratio is greater than one, with a profit greater than zero and the value obtained for the break-even point was less than the production obtained. The research findings allow to conclude that the cultivation of Roselle is profitable given its production characteristics in the area.

Keywords: Cost-Benefit analysis; profits; income; competitiveness.

JEL: M11, M19, M21 


\section{INTRODUCCIÓN}

La flor de Jamaica es una especie vegetal con diferentes propiedades y múltiples usos, originaria de África (SAGARPA, 2018), se le conoce en México como rosa Jamaica, serent, aleluya, y agria de Jamaica (Martínez, 1979) y a nivel internacional como roselle (inglés), I'oiselle (francés), karkade (árabe), bissap (Wolof-Senegal) y dâ (Bambara-Malí), entre otros (McCaleb citado por Contreras y otros, 2009).

La flor de Jamaica es un cultivo que tiene una gran atracción por sus propiedades terapéuticas y múltiples usos. Es consumida tradicionalmente como extracto acuoso para preparar bebidas refrescantes, así como mermeladas, jaleas, licores, harinas para galletas, y muchas más. En los últimos años ha tenido un uso potencial en el área farmacológica debido a los beneficios que produce como medicina alternativa, atribuyéndole propiedades diuréticas, antifebriles, en la disminución del colesterol y la hipertensión. Derivado de lo anterior, se han realizado investigaciones con extractos de Jamaica, demostrando que sus componentes como vitaminas ( $E$ y C), ácidos polifenólicos, flavonoides y antocianinas, poseen actividad antioxidante, contribuyendo a las acciones anticancerígenas y cardioprotectivas (Cid y Guerrero, 2012).

La producción de flor de Jamaica en México se concentra en nueve estados principalmente en el estado de Guerrero y en segundo lugar Oaxaca, en superficie sembrada, la región costa de Oaxaca es la que mayor aporta al estado, especialmente el municipio de Santo Domingo de Morelos (SIAP, 2017), donde el cultivo es una fuente de ingreso importante durante el invierno, siendo beneficiadas 1,100 familias productoras, y fuente de empleo temporal. El presente documento tiene como objetivo analizar la rentabilidad de la producción de flor de Jamaica en Santo Domingo de Morelos, Oaxaca. A continuación, se presenta una revisión de literatura, posteriormente se detalla la metodología y resultados. El documento finaliza con las conclusiones y principales recomendaciones.

\section{MARCO TEÓRICO}

Ibáñez y Caro (2001) reportan indicadores de competitividad a nivel de empresa o unidad económica como el ingreso, el costo, la competitividad precio-costo y la competitividad tasa de ganancia. La competitividad precio-costo, pretende resaltar la capacidad para obtener beneficios entre unidades económicas (países, regiones, empresas) vía la formación de precios de mercado y los costos de producción. Por su parte la competitividad tasa de ganancia o margen de beneficio, determinan la eficiencia en función de los beneficios netos relativos. El análisis económico plantea que los indicadores de rentabilidad son la ganancia, diferencia entre ingreso y costo, la relación beneficio costo y la tasa de rentabilidad.

\section{Producción y tecnología}

En el proceso de la producción, a partir de la combinación de factores de la producción se obtienen determinados productos. Hay una relación entre los factores de la producción (fertilizantes, semillas, mano de obra, agua, etc.) y los productos que se pueden obtener (maíz, trigo, leche, etc.). A partir del estudio de esta relación se obtienen los principios que indica el nivel más adecuado de producción, la cantidad óptima de 
factor empleado, la influencia de los precios sobre la escala de operación, la productividad de los factores, los costos de producción, entre otros.

Por otro lado, tecnología se concibe como el conjunto de conocimientos técnicos, ordenados científicamente, que permiten diseñar y crear bienes y servicios que facilitan la adaptación al medio ambiente y satisfacer las necesidades esenciales y los deseos de las personas. La tecnología en una de sus acepciones más comunes y práctica, se refiere al conjunto de conocimientos, procesos y factores que se requieren para la producción de bienes, los cuales deben combinarse eficientemente para obtener la mayor producción al menor costo. La tecnología está directamente relacionada con los costos y niveles de producción. Finalmente, el caso general de las empresas se relaciona con su comportamiento maximizador de ganancias con diferentes insumos y productos, y que se encuentra sujeta a diversas restricciones en mercados perfectamente competitivos (Varian, 2015). En este sentido, es importante señalar los principales costos que enfrentan las empresas; el costo privado de producción corresponde al precio que tiene que pagar el productor por el uso de los recursos (tierra, trabajo y capital) para llevar a cabo la producción. El costo privado se clasifica en costos explícito e implícito, costos fijo y variable, costos medio y marginal, entre otros. De tal manera, el costo explicito se contabiliza para estimar e incorporar dentro de un sistema contable preestablecido, es decir, son los gastos que efectúa el productor en la compra de los insumos que necesita para llevar a cabo la producción, tales como: costos de la mano de obra, costos de los fertilizantes, costos de las semillas, costos de los insecticidas y herbicidas. Por otro lado, los costos implícitos son gastos fijos a corto plazo que se deben sumar a los costos explícitos con la finalidad de obtener la contabilidad completa de los costos privados de producción.

Los costos fijos totales corresponden a la suma de costos fijos explícitos a corto plazo y los costos implícitos que se incurren, y que se emplean independientemente del nivel de producción. Por otro lado, los costos variables son empleados en un solo ciclo productivo y cambian conforme con el nivel de producción. Finalmente, el costo total corresponde a la sumatoria de aquellos variables y fijos totales.

\section{Costos medio y marginal}

La curva del costo fijo medio se caracteriza porque a medida que aumenta la producción el costo fijo medio disminuye continuamente, esto se debe a que una cantidad constante de costo se divide entre una cantidad de producción cada vez mayor. Por otro lado, el costo marginal se refiere a la adición al costo total imputable a una unidad adicional de producción, lo cual significa que el costo marginal no es más que la cantidad en que se incrementa el costo total dado ante un incremento unitario en la producción.

\section{Ingresos y ganancia}

Los ingresos representan el valor de los bienes y servicios obtenidos con la inversión en una empresa y el valor de los bienes que por efecto de las obras no se destruyen ulteriormente. El incremento en los ingresos proviene de diversas fuentes, dependiendo de las características de cada actividad económica en particular: mayores rendimientos, menores costos, menores pérdidas, entre otros. La ganancia se define como la diferencia que existe entre el ingreso total (IT) y el costo total (CT). Para obtener el ingreso total se 
multiplica la producción por el precio unitario de venta del producto (Pu) y para obtener el costo total se suman todos los costos parciales.

\section{METODOLOGÍA}

\section{Información de campo}

Para llevar a cabo la investigación se obtuvo información general de los productores de Jamaica, localidad, edad, escolaridad, así como del proceso de producción que llevan a cabo, los insumos, mano de obra en cantidad y precio, también la variedad y sus respectivos precios en el mercado; así como superficie sembrada y rendimiento.

Para realizar el trabajo de campo se utilizó el muestreo por conglomerados, se elaboró un cuestionario y se aplicaron encuestas en el municipio de Santo Domingo de Morelos, Oaxaca. Con la información obtenida en el municipio de Santo Domingo de Morelos, se obtuvo el tamaño de la población, un total de 1,748 comuneros reconocidos, donde 1,100 son productores de Jamaica y milpa, se obtuvo un tamaño de muestra de 60 productores distribuidas en 6 localidades del municipio, donde se priorizó las áreas más productoras, el muestreo se desarrolló durante el mes de agosto de 2017. El tamaño de la muestra corresponde al 5\% de la población de productores, es decir, 1100 reconocidos por la SAGARPA. Posteriormente se seleccionaron las seis localidades más importantes. Los elementos de cada localidad fueron escogidas al azar, quedando un tamaño de muestra de 60 productores en total. Los datos proporcionados por los productores que se entrevistaron, fueron procesados en matrices con el fin de calcular los valores parciales, promedios, totales, estructuras porcentuales, entre otros, de las variables tecnología y costos de producción.

\section{Procedimientos de cálculo}

Los procedimientos generales de cálculo utilizados son: valor parcial, valor total y proporción, los cuales se describen a continuación.

Valor parcial. El valor parcial se refiere al valor de una etapa, de una variable o de una parte de un todo en estudio.

Valor total. El valor total se refiere a la sumatoria de los valores parciales de las variables en estudio. El procedimiento de cálculo es: $\mathrm{VT}=\sum \mathrm{VP}$, donde: $\Sigma=$ Sumatoria; $\mathrm{VT}=\mathrm{Valor}$ total; $\mathrm{VP}=$ Valores parciales.

Proporción. La proporción es el valor que representa la participación de un valor parcial con respecto de un total. El procedimiento de cálculo es el siguiente: \% = (VP / VT) * 100, donde: \% = Participación porcentual; VP = Valor parcial; VT = Valor total.

Tecnología. La caracterización de la tecnología requiere de la descripción del proceso de producción, la clasificación de los insumos y la identificación de los coeficientes técnicos de producción. La participación de los insumos, se calcularon dividiendo el valor parcial entre el valor total, el procedimiento de cálculo es:

$$
\%=\frac{V P}{V T} \times 100
$$

Donde: $\mathrm{VT}=$ Valor total de insumo, $\mathrm{VP}=$ Valor parcial del insumo.

Costos de producción. Los costos de producción se calcularon multiplicando la cantidad de insumos utilizados por hectárea por el precio unitario de cada uno de ellos en cada 
una de las etapas de la producción y, finalmente, se sumaron los costos de cada etapa de producción para obtener el costo total. Esto se ilustra a continuación:

$$
\begin{array}{ll}
\mathrm{CPE}=\mathrm{Q}_{i} * \mathrm{P}_{\mathrm{i}} & {[2]} \\
\mathrm{CPT} & =\sum \mathrm{CPE}
\end{array}
$$

Donde: $\sum=$ Sumatoria; $C P E=$ Costo de producción por etapa; $Q_{i}=$ Cantidad de insumo utilizado; $\mathrm{P}_{\mathrm{i}}=$ Precios del insumo; $\mathrm{CPT}=$ Costo de producción total

Estructura de costos. La estructura de costos se obtuvo al dividir el costo de producción por etapa entre el costo total de la producción y multiplicado por cien para obtener el porcentaje.

$$
\% C P E=\frac{C P E}{C P T} * 100
$$

Donde: $\mathrm{CPE}=$ Costo de producción por etapa, $\mathrm{CPT}=$ Costo de producción total

Ingresos. Los ingresos se obtuvieron multiplicando los rendimientos que se obtuvieron por hectárea por el precio unitario del producto en el mercado.

$$
\mathrm{Y}=\mathrm{R} * \mathrm{Pu}
$$

Donde: $\mathrm{Y}=$ Ingreso; $\mathrm{R}=$ Rendimiento; $\mathrm{Pu}=$ Precio unitario del producto en el mercado

Competitividad. Los indicadores de competitividad calculados son: precio-costo y tasa de ganancia. Los procedimientos son los siguientes:

$$
\begin{array}{ll}
\mathrm{RPC}=\mathrm{P} / \mathrm{C} & {[6]} \\
\mathrm{TG}=(\mathrm{P} / \mathrm{C})-1 & {[7]}
\end{array}
$$

Donde: $\mathrm{RPC}=$ Relación precio costo; $\mathrm{TG}=$ Tasa de ganancia; $\mathrm{P}=$ precio; $\mathrm{C}=$ Costo

\section{RESULTADOS}

\section{Costos de producción por hectárea}

El costo total es de $\$ 16,651.22$ pesos por hectárea, identificando que el $9 \%$ se debe a los costos fijos, de $\$ 1,472.51$ pesos y el $91 \%$ es de costos variables de $\$ 15,178.71$ pesos.

Tabla 1. Costos fijos y variables de la producción de flor de Jamaica en Santo Domingo de Morelos (MXN)

\begin{tabular}{lcc}
\hline \multicolumn{1}{c}{ Concepto } & Total & $\%$ \\
\hline Costos fijos & $1,472.5$ & 8.8 \\
Renta del terreno & $1,421.4$ & 8.5 \\
Transporte & 51.1 & 0.3 \\
Costos variables & $15,178.7$ & 91.2 \\
Siembra & $1,022.2$ & 6.1 \\
Control de maleza & $2,945.8$ & 17.7 \\
Control de plagas & 124.2 & 0.7 \\
Cosecha & $10,660.0$ & 64.0 \\
Secado & 275.3 & 1.7 \\
Empaque & 151.3 & 0.9 \\
Costo total & $16,651.2$ & 100.0 \\
\hline
\end{tabular}

Fuente. Elaborado con datos de encuesta, 2017

El rendimiento promedio por hectárea, a nivel municipio, es de 200.64 kg por hectárea de Flor de Jamaica seca, valor que se encuentra por debajo del rendimiento medio a nivel 
nacional, de $310 \mathrm{~kg}$ de flor seca. El precio cambia de acuerdo a la variedad de Flor de Jamaica, en los últimos dos años se ha mantenido la Flor de Jamaica criolla a \$100.00 pesos el kilo, cuando en el 2014 estaba a $\$ 70.00$ pesos el $\mathrm{kg}$. Mientras que la Flor de Jamaica tempranera tuvo un precio de $\$ 70.00$ pesos el $\mathrm{kg}$ en el 2015 y 2016 y en el 2014 tenía un precio de $\$ 50.00$ pesos.

La diferencia en el precio radica en la calidad del producto, la flor de Jamaica criolla tiene mayor apreciación por la acidez en comparación de la tempranera que tiene más color que acidez.

Tabla 2. Precio de Flor de Jamaica en Santo Domingo de Morelos

\begin{tabular}{lccc}
\hline Variedad & 2014 & 2015 & 2016 \\
\hline Criollo & 70 & 100 & 100 \\
Tempranera & 50 & 70 & 70 \\
\hline
\end{tabular}

Fuente. Elaborado con datos de encuesta, 2017.

Tomando en cuenta a la Flor de Jamaica criolla, con un rendimiento de $200.64 \mathrm{~kg}$, a un precio de $\$ 100.00$ pesos, se tiene un ingreso de $\$ 20,063.68$ pesos por hectárea.

Tabla 3. Ingreso promedio en Santo Domingo de Morelos

\begin{tabular}{cccc}
\hline & $\begin{array}{c}\text { Rendimiento } \\
(\mathrm{kg})\end{array}$ & $\begin{array}{c}\text { Precio } \\
(\$)\end{array}$ & $\begin{array}{c}\text { Ingreso } \\
(\$)\end{array}$ \\
\hline Jamaica criolla & 200.64 & 100.00 & $20,063.68$ \\
\hline \multicolumn{4}{c}{ Fuente. Elaborado con datos de encuesta, 2017. }
\end{tabular}

\section{Punto de equilibrio}

El punto de equilibrio no es una técnica para evaluar la rentabilidad de una inversión, sino que es una importante referencia para conocer las relaciones entre los costos fijos, los costos variables y los beneficios. Es el nivel de producción en el que son iguales los beneficios por venta a la suma de los costos fijos y los variables. Para el cultivo de Jamaica el nivel de producción donde no hay perdidas ni ganancias es cuando se produce 60.48 $\mathrm{kg}$ de flor seca, lo que equivaldría a un valor de $\$ 6,047.92$ pesos por hectárea.

Figura 1. Punto de equilibrio.

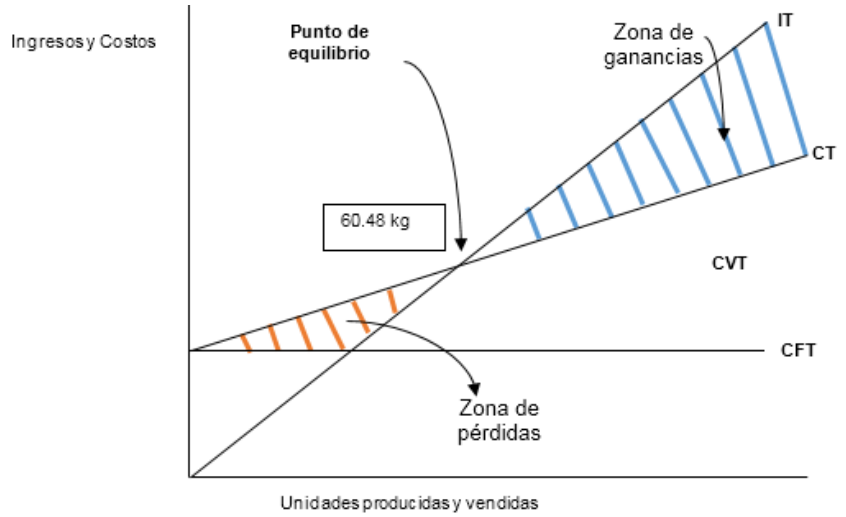

\section{Ganancia y relación beneficio-costo}

Con el costo total de producción de $\$ 16,651.22$ pesos y un ingreso de $\$ 20,063.68$ pesos, se tiene una ganancia de $\$ 3,412.46$ pesos por hectárea. 
Tabla 4. Ganancia promedio de Jamaica en Santo Domingo de Morelos

Ingreso

Costo total

Ganancia

$(\$)$

(\$)

$\frac{16,651.22}{3,412.46}$

La relación beneficio-costo genera un valor de 1.20, significa que en la producción se recupera todo lo invertido y se obtiene una rentabilidad de 20 centavos por cada peso invertido, es decir aun con las condiciones actuales de producción, en el municipio de Santo Domingo de Morelos, es viable la producción de Flor de Jamaica, no solo por cuestión económica, sino social porque es una manera de mantener viva el cultivo en la región y generar empleo temporal, además de beneficiar a los pequeños productores sin mencionar el encadenamiento productivo.

Tabla 5. Indicadores de rentabilidad en Santo Domingo de Morelos

\begin{tabular}{lr}
\hline Conceptos & \multicolumn{1}{c}{ Valores } \\
\hline Ingreso & $\$ 20,063.68$ \\
Costo & $\$ 16,651.22$ \\
Ganancia & $\$ 3,412.46$ \\
Relación B/C & 1.20 \\
Punto de equilibrio $(\mathrm{kg})$ & $60.48 \mathrm{~kg}$ \\
\hline
\end{tabular}

Fuente. Elaborado con datos de encuesta, 2017.

\section{CONCLUSIONES}

En el municipio de Santo Domingo de Morelos (Oaxaca), la producción de Flor de Jamaica tiene indicadores como relación beneficio-costo y ganancia, mayores a su punto crítico. A pesar de las condiciones actuales de producción, favorece a la economía familiar. No beneficia solamente a las familias productoras, sino que también a otros pobladores del municipio por ofrecer empleo temporal durante los meses de noviembre y diciembre de cada año. Así mismo, existen oportunidades de industrializar el producto buscando aumentar el índice de bienestar y que la actividad económica genere empleos de mayor productividad. Por lo tanto, y con base a los resultados obtenidos, se puede afirmar que la producción de Flor de Jamaica es rentable, debido a que el costo de producción es menor a los ingresos. Su producción permitirá generar ingresos importantes sobre todo para los pequeños productores agrícolas de la zona.

\section{REFERENCIAS}

Ariza, R., Serrano, V., Navarro, S., Ovando, M., Vasquez, E., Barrios, A., Michel, A., Guzman, S., Otero, M. A. (2014). Variedades mexicanas de Jamaica (Hibuscus sabdariffa L.) 'Alma Blanca' y 'Rosaliz' de color claro, y 'Cotzaltin' y 'Tecoanapa' de color rojo. Revista fitotecnia mexicana, 37, 2.

Arteaga, R. (2017). Comercialización internacional de la producción de Jamaica del municipio de Aquila, Michoacán. Instituto de Investigaciones Económicas y Empresariales, Universidad Michoacana de San Nicolás de Hidalgo. Disponible en: http://bibliotecavirtual.dgb.umich.mx:8083/xmlui/handle/DGB UMICH/1044

Cid, S., Guerrero, J. (2012). Propiedades funcionales de la Jamaica (Hibiscus sabdariffa L.) En Temas Selectos de Ingeniería de alimentos (TSIA) 6-2(2012). UDLAP, México.

Contreras G., Soto, J., Huchin, A. (2009). Tecnología para el cultivo de Jamaica en Quintana Roo. INIFAP. Folleto técnico No. 13. Quintana Roo, México.

Caro, J., Ibañez, C. (2001). Algunas teorías e instrumentos para el análisis de la competitividad. IICA, San José, Costa Rica. Disponible en: https://agris.fao.org/agrissearch/search.do?recordID=XL2012000912 
Duarte, Z., Zamora, V., Montalvo, E., Sáyago, S. (2016). Caracterización nutricional de 20 variedades mejoradas de Jamaica (Hibiscus sabdariffa L.) cultivadas en Mexico. Revista fitotecnia mexicana, $39,3$.

Escorcia, M., Escoto, H., Espinoza, K. (2007). Aporte económico de la producción de vino de flor de Jamaica en Chinantlan del municipio de Chinandega en el periodo 2005-2006. Universidad Nacional Autónoma de Nicaragua. Disponible en: http://riul.unanleon.edu.ni:8080/jspui/retrieve/4691

López, G., Sumaya, M., Jiménez, E., Balois, R., Medina, R., Guzmán, J. (2017). Propiedades antimicrobianas y antioxidantes de Jamaica. Acta Agrícola y Pecuaria, 3(3), 61-69.

Nicholls, J., Ramírez, M. (2014). Usos y aplicaciones medicinales e industriales de la flor de Jamaica. Universidad Nacional Abierta y a Distancia UNAD. Disponible en: https://repository.unad.edu.co/handle/10596/2727

Secretaría de Agricultura, Ganadería, Desarrollo Rural, Pesca y Alimentación (SAGARPA). (2018): Conoce más sobre la Flor de Jamaica. SAGARPA, México. En línea, disponible en: https://www.gob.mx/sagarpa/articulos/conoce-mas-sobre-la-flor-de-jamaica

SIAP, (2017): Anuario Estadístico de la Producción Agrícola. Disponible en: http://nube.siap.gob.mx/cierre agricola/

Varian, H. (2015). Microeconomía Intermedia. Editorial Antoni Bosch. 